\title{
Encapsulación de la pulpa de camu camu (Myciaria dubia) y deshidratado de las cápsulas
}

\section{Encapsulation of the pulp of camu camu (Myciaria dubia) and dehydrated capsules}

\author{
Víctor Manuel Terry Calderón $\square_{y}$ iD Oscar Osso
}

Universidad Le Cordon Bleu. Lima, Perú

\begin{tabular}{llll}
\hline Recibido: 12/04/2018 & Revisado: 02/04/2019 & Aceptado: 10/06/2019 Publicado: 30/06/2019
\end{tabular}

\section{RESUMEN}

El objetivo de este trabajo fue estudiar la formación del encapsulado de la pulpa de camu camu en una matriz de alginato de calcio utilizando el método de goteo por inmersión de una solución de alginato de sodio, seguido de una operación de secado por arrastre.

El proceso de gelificación externa ocurre con la difusión del ion calcio desde una fuente que rodea al hidrocoloide hacia la solución de alginato de pH neutro. La formación del gel se inicia en la interface y avanza hacia el interior a medida que la superficie se encuentra saturada de iones calcio, de manera que el ion sodio proveniente de la sal de alginato es desplazado por el catión divalente solubilizado en agua.

Este interacciona con los G-bloques de diferentes moléculas poliméricas, enlazándolas entre sí. Aunque, la fuente de calcio más usada ha sido el $\mathrm{CaCl}_{2}$ debido a su mayor porcentaje de calcio disponible, existen otras sales empleadas con menor frecuencia tales como el acetato monohidratado y el lactato de calcio.

La pulpa de camu camu refinada y a un $\mathrm{pH}$ 3,3 neutralizada con bicarbonato de sodio hasta un $\mathrm{pH}$ 4,6 y utilizando la gelificación directa con alginato de sodio en una proporción de 1g/100g y aplicando la técnica del goteo en solución de cloruro de calcio 1g/100g Y después de varios ensayos se obtuvo la técnica para producir las esferas de camu camu las cuales fueron tamizadas y deshidratadas a una temperatura de $60{ }^{\circ} \mathrm{C}$ obteniéndose $7,40 \mathrm{~g} / 100 \mathrm{~g}$ de pulpa de camu camu.

Palabras clave: camu camu, esferificación de camu camu, deshidratación del camu camu.

\section{ABSTRACT}

The objective of this work was to study the formation of the pulp of camu camu in a matrix of calcium alginate encapsulation using the drip method by immersion of alginate solution of sodium, followed by a drying operation by dragging the process of external Gelation occurs with the calcium ion diffusion from a source that surrounds the hydrocolloid towards neutral $\mathrm{pH}$ alginate solution. Gel formation begins in the interface and moves inside to the surface is saturated with calcium ions, so that from the salt of alginate sodium ion is displaced by the divalent cation solubilized in water. 
This interacts with the G- blocks of different polymer molecules, linking them together. Although, the most widely used source of calcium has been $\mathrm{CaCl}_{2}$ due to its higher percentage of available calcium, there are other salts employed less frequently such as acetate monohydrate and calcium lactate.

Pulp of camu camu refined and at $\mathrm{pH} 3.3$ neutralized with baking soda to $\mathrm{pH} 4.6$ and using the direct gelling with sodium alginate in a proportion of $1 \mathrm{~g} / 100 \mathrm{~g}$ and applying the drip technique in solution of $1 \mathrm{~g}$ calcium chloride / $100 \mathrm{~g}$. And after several trials was the technique to produce areas of camu camu which were sifted and dried at a temperature of $60{ }^{\circ} \mathrm{C}$ resulting in $7,40 \mathrm{~g}$ / $100 \mathrm{~g}$ of pulp camu camu.

Keywords: camu camu, spherification of camu camu, dehydration of camu camu.

\section{INTRODUCCIÓN}

El proceso de gelificación externa ocurre con la difusión del ion calcio desde una fuente que rodea al hidrocoloide hacia la solución de alginato de $\mathrm{pH}$ neutro.

La formación del gel se inicia en la interfase y avanza hacia el interior a medida que la superficie se encuentra saturada de iones calcio, demanera queelion sodio proveniente de la sal de alginato es desplazado por el catión divalente solubilizado en agua.

Este interacciona con los G-bloques de diferentes moléculas poliméricas, enlazándolas entre sí. Aunque, la fuente de calcio más usada ha sido el $\mathrm{CaCl}_{2}$ debido a su mayor porcentaje de calcio disponible, existen otras sales empleadas con menor frecuencia tales como el acetato monohidratado y el lactato de calcio. Revista Venezolana de Ciencia y Tecnología de Alimentos (2008).

Los alginatos son reconocidos como inocuos y seguros según la Food and Drug Administration (FDA) de los Estados Unidos, están listados en el Codex y en la World Heald Organization (WHO) de las naciones Unidas donde se establece que la ingesta diaria para los humanos de $50 \mathrm{mg} / \mathrm{kg}$ de peso corporal. Avendaño C (2013)
Alginato sódico (C6H706Na), también llamada algina, es un tipo de electrolito de macromolécula orgánica entre algas de tipos de algas marrones, tales como algas, como componentes principales de la membrana de la célula con otras sustancias.

Los componentes químicos de alginato de sodio pertenecen a los hidratos de carbono y que solo contiene carboxilo libre (-COONa) en contraste con el almidón y la fibrina.

Al mismo tiempo, es un tipo de macromolécula heteropolimero cadena tipo interpuesto por copolimerización entre el ácido manurónico y guluronicacido.

Fórmula molecular: $\mathrm{C} 6 \mathrm{H} 706 \mathrm{Na}$ peso molecular: 216, Alginate Industry (2013).

El Camu-camu (Myrciaria dubia H.B.K.) es de gran importancia comercial por su alto contenido de vitamina C (ácido ascórbico), de aproximadamente 2800 mg/100g de pulpa fresca, concentración ostensiblemente superior al de otros frutos (naranja con 92 mg/100g de pulpa y limón con $44,2 \mathrm{mg} / 100 \mathrm{~g}$ de pulpa) y hortalizas del mundo, lo que le confiere gran potencial económico en la agroindustria, pues se sabe según Villachica (2008), que la vitamina 
C interviene en la totalidad de nuestros metabolismos: síntesis del colágeno, síntesis hormonales, estimulación de la cicatrización y propiedades antioxidantes (Ramos et al., 2005).

En nuestro país existen una gran variedad de alimentos funcionales que no son explotados, cuyo conocimiento para aplicación tecnológica son casi desconocidos, entre ellos se encuentra el Myciaria dubia "Camu camu" por tener un gran contenido de vitamina $\mathrm{C}$, que crece en la zona amazónica del Perú, el fruto es de forma esférica de color rojiza y su parte comestible corresponde al mesocarpio (Pinedo y Armas, 2007).

Sin embargo, la inestabilidad de la vitamina en la actualidad es considerada el principal "problema" dentro de la cadena de producción de pulpa. Pues la vitamina $C$, es probablemente la menos estable de las vitaminas hidrosolubles. Durante los últimos años se ha observado un interés sobre las propiedades del alginato en la industria alimentaria (Gallegos, 2015).

Los alginatos son extraídos principalmente de tres especies de algas marrones; estas incluyen Laminaria hyperborea, Ascorphyllum nodosum y Macrocystis pyrifera (Avendaño, López y Palou, 2013) Estos tienen propiedades para formar geles y soluciones altamente viscosas (Hernández et al., 2011).

En cuanto a la capacidad gelificante de los alginatos, la formación del gel se inicia a partir de una solución de sal de alginato y una fuente de calcio externa o interna, donde el ion calcio se difunde hasta alcanzar la cadena polimérica y resultando un material sólido con características de un gel (Funami et al., 2009).
Estas ventajas del alginato y las recientes tendencias tecnológicas se han fusionado en la técnica de encapsulación, resultando en un producto final que permite proteger a los compuestos encapsulados de factores adversos mejorando así su estabilidad y biodisponibilidad (Gallegos, 2015).

El presente trabajo de investigación tiene por objetivo evaluar la proporción del polímero orgánico requerido para realizar la esferificación de la pulpa Myciaria dubia "camu camu" y la tecnología apropiada para su deshidratación.

\section{MATERIALES Y MÉTODOS}

El trabajo fue realizado en el laboratorio de la universidad Le Cordon Bleu en la ciudad de Lima, durante los meses de marzo a diciembre del año 2018

\section{Equipos}

Balanza de precisión de 200 g a 3000 g; 0,001 g, Refinadora, pulpeadora.

- Refractómetro de 0 a 40 Brix, secador de aire caliente, equipo de refrigeración y congelación, termómetro 0 a $20^{\circ} \mathrm{C}$.

\section{Material de laboratorio}

- Bureta de $50 \mathrm{ml}$ (2), pipetas (3), Erlenmeyer de $250 \mathrm{ml}$ (3), embudo, pipeta automática $\mathrm{P}-1000$ y probeta de $100 \mathrm{ml}$.

\section{Reactivos}

- Alginato de sodio (Gelfin) con un código E-401, gelificante espesante de alginato de sodio. Cloruro de calcio, solución de Lugol, solución de vitamina C y solución de almidón soluble.

Determinación de la vitamina C por el método de iodometría. 


\section{RESULTADOS}

Características de la materia prima:

La tabla 1 muestra el estado de madurez del

fruto para su debida calificación.

Tabla 1. Estado de madurez sensorial del camu camu

\begin{tabular}{ll}
\hline & Estado de madurez: \\
& Coloración de la \\
Clase & cáscara
\end{tabular}

1

2

$75 \%$

3

$50 \%$

4

$25 \%$

5

$100 \%$
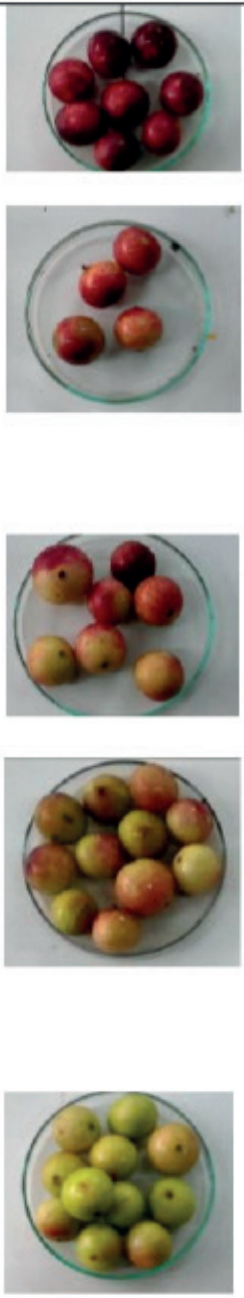
Tabla 2. Características del estado de madurez del material

\begin{tabular}{cccc}
\hline Estado de madurez & Color de la cáscara & $\begin{array}{l}\text { Aspecto del } \\
\text { mesocarpio }\end{array}$ & Sabor \\
\hline Maduro & Rojo (75\%) & Incoloro translúcido & Ácido \\
\hline
\end{tabular}

Relación peso de pulpa/peso de materia prima $(\mathrm{kg} / \mathrm{kg})$ se muestra en el siguiente cuadro:

Tabla 3. Las características del camu camu se muestran en el siguiente cuadro (Grados Brix, Concentración de vitamina $\mathrm{C}$ en la pulpa y $\mathrm{pH}$ )

\begin{tabular}{cc}
\hline $\mathrm{N}$ & $\begin{array}{c}\mathrm{kg} \text { de pulpa/kg } \\
\text { de materia } \\
\text { prima }\end{array}$ \\
\hline 1 & 0,467 \\
2 & 0,397 \\
3 & 0,406 \\
\hline
\end{tabular}

Valor del pH, oBrix y contenido de vitamina $\mathrm{C}$ en la pulpa de camu camu:

Tabla 4. Características de pulpa de camu camu

\begin{tabular}{cccc}
\hline $\mathrm{N}$ & ${ }^{\circ}$ Brix & $\begin{array}{c}\text { Concentración } \\
\text { de vitamina } \mathrm{c} \\
\mathrm{mg} / 100 \mathrm{ml}\end{array}$ \\
\hline 1 & 6,7 & 1480,00 & 3,1 \\
2 & 6,9 & 1520,00 & 3.7 \\
4 & 5,9 & 1350,00 & 2.9 \\
\hline
\end{tabular}


Desarrollo de la tecnología de esferificación y deshidratación de la pulpa de camu camu Se muestra la secuencia de operaciones para definir la tecnología de esferificación de camu camu deshidratados, después de varios ensayos este fue el resultado de

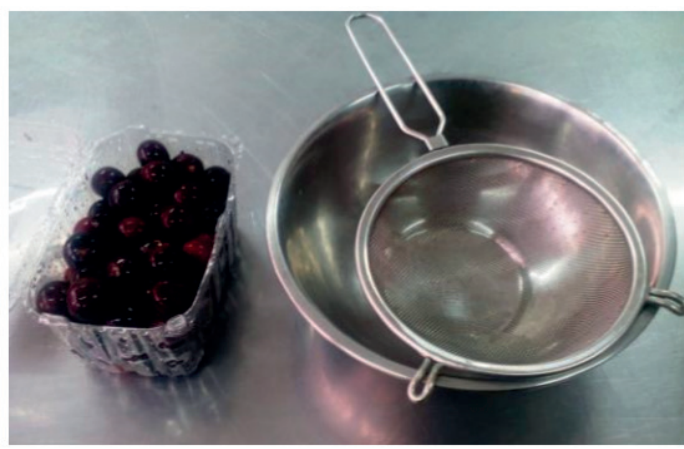

Figura 1. Recepción de camu camu

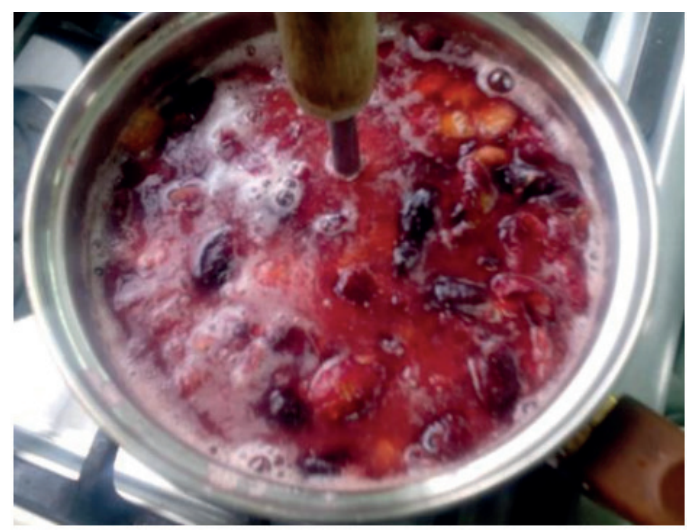

Figura 3. Segunda extracción

La pulpa separada de los residuos (fig. 5) es colocada en tubos de centrifugación (fig. 6) y dichas investigaciones El camu camu fue recepcionado, pesado y lavado como se aprecia en la foto, donde el camu camu fue pulpeado y realiza la extracción de la pulpa (figuras 1, 2, 3 y 4).

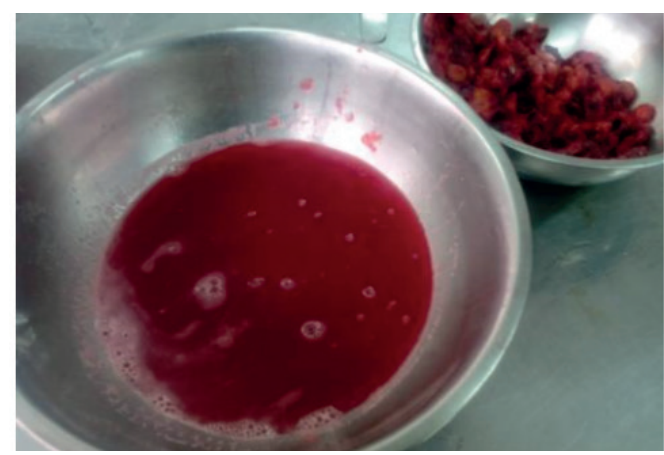

Figura 2. Primera extracción de pulpa

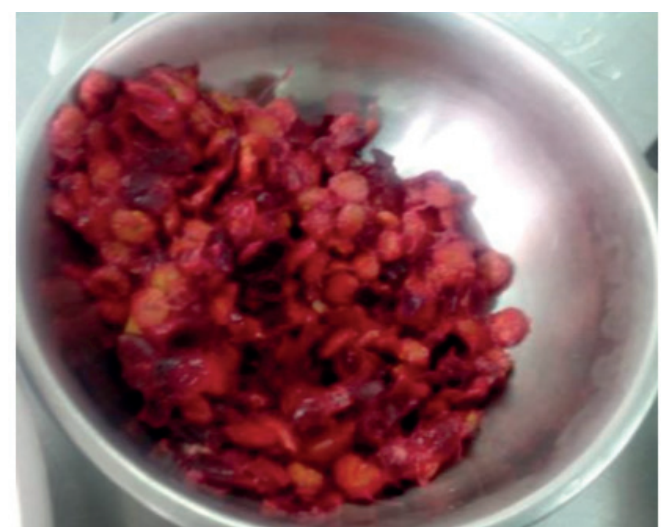

Figura 4. Residuos del pulpeo

en la fig. 7 se aprecia la separación de la pulpa refinada en los tubos de centrifuga. 


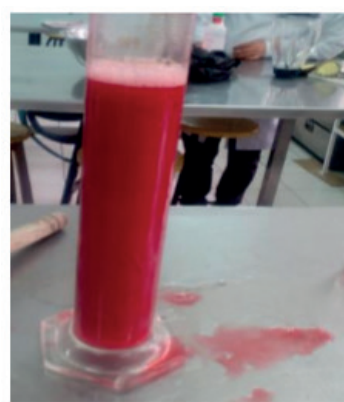

Figura 5. Pulpa sin refinar

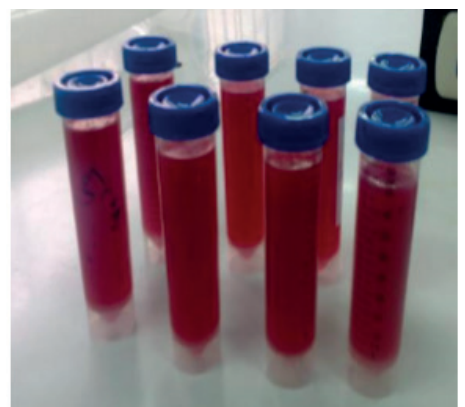

Figura 6. Pulpa a centrifugación

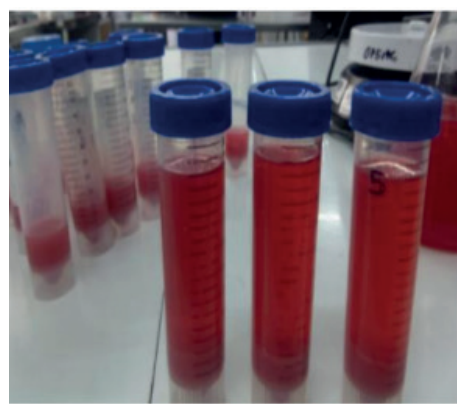

Figura 7. Pulpa refinada
En la figura 8, se observa la pulpa refinada y los residuos sólidos, en la figura 9 la pulpa refinada y pasteurizada

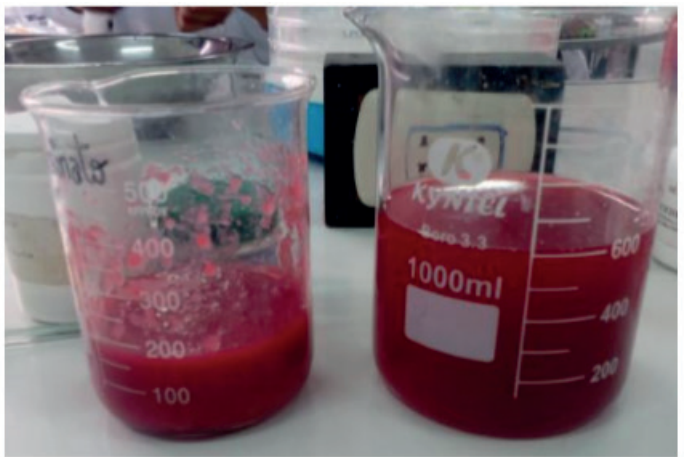

Figura 8. Pulpa refinada y residuos

En la fig. 10 se muestran los reactivos utilizados para la esferificación, el Alginato de sodio, el cloruro de calcio y el acetato de sodio.

En el figura 11 se observa el proceso de formación de esferas utilizando una bureta es colocada en envases flexibles y sometidos congelación para sus usos posteriores:

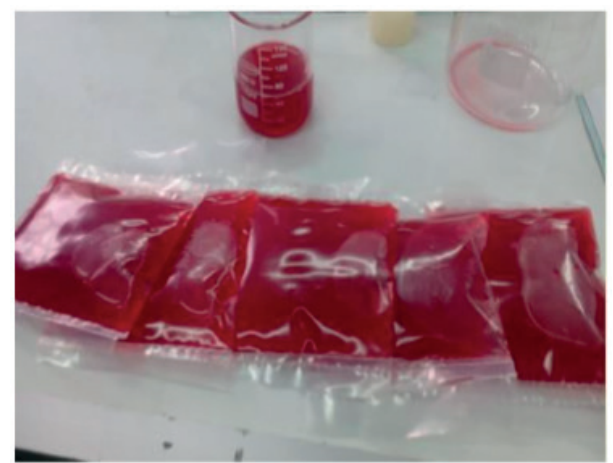

Figura 9. Pulpa envasada y congelada $a-5^{\circ} \mathrm{C}$

que permite que la mezcla de pulpa refinada de camu camu y Alginato de sodio pueda ser colocada gota a gota en el beaker que contiene cloruro de calcio, en la figura 12 se puede apreciar las esferas formada por la pulpa de camu camu.

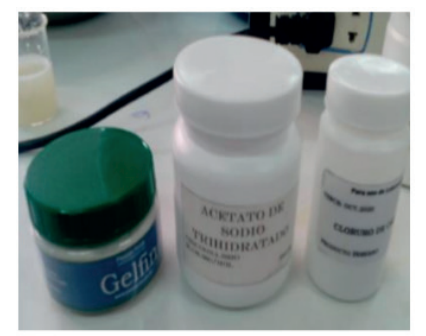

Figura 10. Reactivos para la esferificación

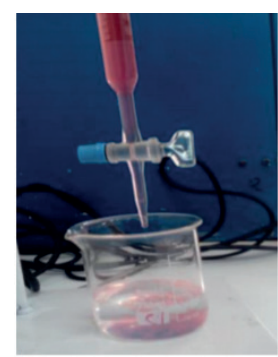

Figura 11. Formación de esferas de la pulpa

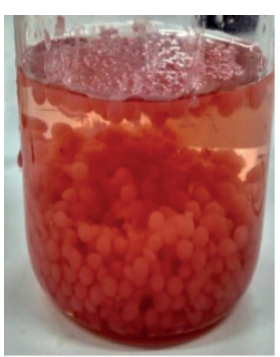

Figura 12. Esferas de camu camu 


\section{Operación de esferificacion de la pulpa de camu camu}

Caracterización de la muestra y la base de cálculo para elaboración del producto

Tabla 5. Base de cálculo: 105,34 g

\begin{tabular}{ll}
\hline Peso de camu camu & $105,34 \mathrm{~g}$ \\
Volumen & $105,00 \mathrm{ml}$ \\
$\mathrm{pH}$ & 3,18 \\
oBrix & 6 \\
Vitamina C & $21,33 \mathrm{mg} \mathrm{VitC/g}$ de pulpa de camu camu \\
\hline
\end{tabular}

Estando la pulpa con un pH bajo se hizo necesario la neutralización hasta un pH de 4,5 a 4,7:

Tabla 6. Resultado de la neutralización de la pulpa de camu camu

\begin{tabular}{ll}
\hline Peso total de bicarbonato de $\mathrm{Na}$ & $0,9 \mathrm{~g}$ \\
$\mathrm{pH}$ & 4,69 \\
oBrix & 6,5 \\
\hline
\end{tabular}

El alginato de sodio al adicionarse en agua forma un coloide con las siguientes propiedades:

Tabla 7. Preparación de la Solución coloidal de alginato de sodio

\begin{tabular}{ll}
\hline Volumen & $100 \mathrm{ml}$ \\
Alginato de sodio & $2 \mathrm{~g} / 100 \mathrm{l}$ \\
$\mathrm{pH}$ & 7,84 \\
Solidos solubles & 2,00 \\
\hline
\end{tabular}

A fin de forma la matriz de alginato y pulpa de camu camu se mezclan ambas soluciones:

Tabla 8. Adición del Alginato de sodio a la pulpa neutra de camu camu

\begin{tabular}{ll}
\hline Volumen total & $200 \mathrm{ml}$ \\
Solidos solubles & 4,00 \\
$\mathrm{pH}$ & 4,99 \\
\hline
\end{tabular}


La solución de cloruro de calcio que sirve para formar la película en torno a la esfera:

Tabla 9. Preparación de solución de cloruro de calcio

\begin{tabular}{ll}
\hline Volumen & $200 \mathrm{ml}$ \\
Cloruro de calcio & $1 \mathrm{~g} / 200 \mathrm{ml}$ \\
$\mathrm{pH}$ & 5,96 \\
\hline
\end{tabular}

Mezcla de cloruro de calcio y solución coloidal de cloruro de calcio con alginato de sodio:

Tabla 10. Esferificación del camu camu en solución con el alginato de sodio adicionado a la solución de cloruro de calcio

\begin{tabular}{ll}
\hline Peso del material encapsulado & $135,12 \mathrm{~g}$ \\
Volumen residuos de cloruro de calcio & $240 \mathrm{ml}$ \\
$\%$ de sólidos de las esferas & $6 \%$ \\
$\%$ de agua de las esferas & $94 \%$ \\
\hline
\end{tabular}

Resultado de la deshidratación de las esferas de camu camu:

Tabla 11. Esferas de pulpa de camu cam deshidratado

\begin{tabular}{ll}
\hline Peso deshidratado & $7,70 \mathrm{~g}$ \\
Vitamina C & $71,08 \mathrm{mg}$ de Vit. C/g de esfera \\
& deshidratada \\
\hline
\end{tabular}

Determinación del contenido de vitamina C en el líquido residual:

Tabla 12. Contenido de vitamina $\mathrm{C}$ residual en la solución de cloruro de calcio

\begin{tabular}{ll}
\hline Volumen & $240 \mathrm{ml}$ \\
Vitamina C & $6,66 \mathrm{mg} \mathrm{Vit} \mathrm{C/ml} \mathrm{de} \mathrm{solución} \mathrm{Cl}_{2} \mathrm{Ca}$ \\
\hline
\end{tabular}


Secado de las capsulas al arrastre con aire. Temperatura de secado con aire caliente: $60^{\circ} \mathrm{C}, \mathrm{y}$ HRE: $70 \%$ :

Tabla 13. Record de deshidratación de las esferas de camu camu encapsuladas

\begin{tabular}{cc}
\hline Tiempo $(\mathbf{t}) \mathbf{h}$ & Peso $(\mathbf{W}) \mathbf{g}$ \\
\hline 0 & 135.12 \\
1 & 85.40 \\
2 & 50.30 \\
3 & 35.11 \\
4 & 23.75 \\
5 & 13.48 \\
6 & 7.72 \\
\hline
\end{tabular}

En las figuras 13 y 14 las esferas fueron colocadas en un secado de arrastre de aire a una temperatura de $60^{\circ} \mathrm{C}$ y en la figura 15 se muestran ya las esferas deshidratadas.

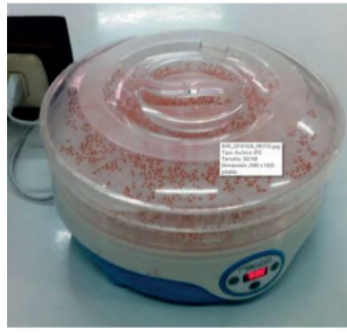

Figura 13. Secado de la esferas

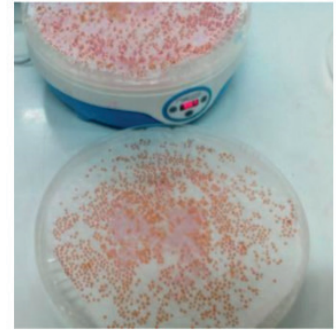

Figura 14. Esferas secadas

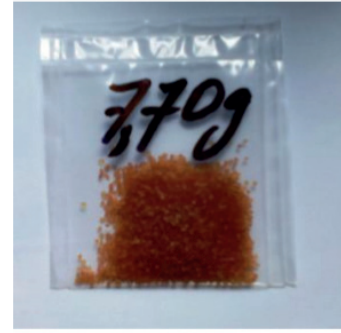

Figura 15. Esferas envasadas

La elaboración de la curva de secado de las cápsulas se realizó en un secador de aire caliente por arrastre a temperatura de $60^{\circ} \mathrm{C}$.

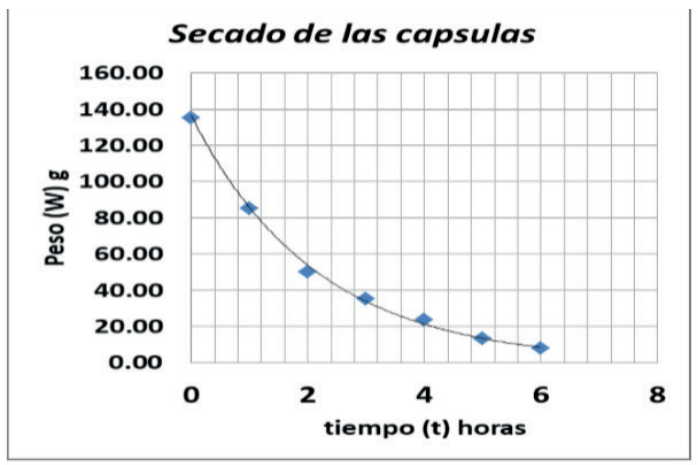

Figura 16. La curva de secado 
Donde g: Peso (w) en gramos residual y t: el tiempo de secado en horas.

Por análisis de regresión se obtiene la siguiente expresión:

$$
W=136,5 \cdot e^{-0.465(t)}
$$

Con un coeficiente de correlación R2=0,9958 En el gráfico se puede observar que las dos primeras horas sigue un curso constante de pérdida de peso, que equivale a la pérdida de humedad y de acuerdo a la tabla tiene un valor de 84,82 gramos, en términos de porcentaje equivale a reducción en peso de 62,77 \%.

Esta fase se conoce como secado a razón constante y está representado por una línea recta. , para posteriormente esta velocidad de pérdida de peso va siendo variable a partir de la segunda hora.

\section{DISCUSIÓN}

Según Gallegos (2015), la encapsulación es una técnica de obtención de una barrera que retarda las reacciones químicas con el medio que lo rodea promoviendo un aumento en la vida útil del producto, incluso facilitando su manipulación.

Las cápsulas pueden tener forma esférica o irregular, constituidas por una membrana simple, múltiples capas e incluso núcleos múltiples.

La forma de las cápsulas obtenidas fue esférica, utilizando el alginato sodio y cloruro de calcio para su formación, de esta manera se obtuvo zumo de camu camu (Myciaria dubia) encapsulado, logrando de esta manera una fácil manipulación de vitamina $\mathrm{C}$ el cual se encuentra en el zumo concordando de esta manera lo expuesto por el autor.
Según Madene et al., 2006, en cuanto a la técnica de deshidratación atómica el tamaño de partículas de los encapsulados varia de 0,3 a 10 mm, así como algunas ventajas de esta técnica son: permite una distribución de tamaños específicos, baja porosidad, altas velocidades de secado, optimas velocidades de transferencia de calor y masa, áreas más pequeñas de flujo, alta eficiencia térmica, bajo presupuesto y costos de mantenimiento, así como un fácil control.

La cantidad utilizada de alginato y cloruro de sodio fue de $1 \mathrm{~g} / 100 \mathrm{~g}$ lo cual nos resultó aceptable a la textura y forma de la esfera de camu camu, nuestra cantidad utilizada no coincide con lo citado por los autores, lo cual nos indicaría que la cantidad de aditivos a utilizar para la esferificacion depende también de la materia prima que se desea esterificar, y un factor importante fue el $\mathrm{pH}$ que debe tener un mínimo de 4,7.

\section{CONCLUSIONES}

El alginato es un polímero adecuado para la encapsulación por ser bio compatible, no tóxico y degradable.

En el trabajo de investigación la cantidad de 1g/ $100 \mathrm{~g}, \mathrm{pH}$ 7,2 de alginato fue aceptada para la formación de la esferificación de camu camu. Utilizando como adyuvante de formación de esferas o cápsulas al cloruro de calcio a 1g/100 g, obteniéndose un material deshidratado de 7,7 gramos / $100 \mathrm{~g}$ de pulpa.

Diversas investigaciones demostraron que los compuestos encapsulados se encuentran biodisponibles y por tanto pueden mantener sus actividades funcionales durante el procesamiento y almacenaje de los alimentos.

La tecnología definida en el presente trabajo con la deshidratación por secado de aire 
caliente resultó el más adecuado y favorece la conservación del producto.

\section{REVISIÓN BIBLIOGRAFICA}

Avendaño C. (2013). Propiedades del alginato y aplicaciónes en alimentos. Temas selectos de Ingenieria de los alimentos 7-1(2013):8796 Puebla, México.

Alginate Industry (2013). Recuperado en el 15 diciembre 2018 de Propiedades del Alginato de sodio, https://www.iroalginate.com/sp/ Alginates/Sodium-Alginate.htm

Funami, T., Fang, Y., Noda, S., Ishihara, S., Nakauma., Draget, K., y Phillips, G. (2009) Propiedades reológicas del alginato de sodio en un sistema acuoso durante la gelificación en relación con las estructuras supramoleculares y la unión de Ca2 +. Alimentos hidrocoloides. 23 (7), 17461756.

Gallegos, G. M. (2015) Utilización de Alginato de Sodio para la encapsulación de complejo B. Revista de Ciencia y Tecnología. 6 (1), 120.

Hernández, Carmona. G., Rodríguez, M. Y., Arvizu, H. D., Reyes, T. R., Murillo, A.J., y Muñoz, O. M. (2011). Avances tecnológicos en la producción de alginatos en México. Scielo. (13), $52-55$.

Madene, A., Jacquot, M., Sher, J., y Desobry, S. (2006). Encapsulación de sabor y liberación controlada, revisada en; International Journal of Food Science and Technology. 41: 1-21.

Pinedo, (2007). El camu camu y sus usos populares como planta medicinal. Revista de Agroecología. LEISA 23.3. Diciembre 2007. Universidad Nacional de la Amazonia Peruana - UANP, Iquitos, Perú
Ramos et al. (2005). Evaluación de factores de procesamiento y conservación de pulpa de Myrciaria dubia H.B.K. (camu-camu) que reducen el contenido de vitamina (ácido ascórbico). Revista Amazónica de Investigación Alimentaria, v.2 n² p. 89 - 99 (2002) Facultad de Ingeniería en Industrias Alimentarias UNAP, Iquitos, Perú.

Ramos, Z., Panduro, L., y Panduro, M. (2002). Evaluación de factores de procesamiento y conservación de pulpa de Myrciaria dubia h.b.k. (camu-camu) que reducen el 22 contenido de vitamina (ácido ascórbico). Revista Amazónica de Investigación Alimentaria. 2 (2), 89 - 99.

Salinas-Hernández (2007). Modelación del deterioro de productos vegetales frescos cortados. División Académica de Ciencias Agropecuarias. UJAT. (GAGA) Centro de Investigación en Alimentación y Desarrollo, A.C. Carretera a La Victoria $0,6 \mathrm{~km}$. C. P. 83000, Hermosillo, Sonora, México. (MEP) Instituto de Tecnología de Alimentos-UNL, Argentina. (FUM) División Académica de Ciencias Básicas. UJAT.

Villachica (2008). El cultivo de camu camu en la Amazonia Peruana, tratado de Cooperación Amazónica, secretaria Pro Tempore, Lima, Perú. Pag 3-7.

Madene, A., Jacquot, M., Sher, J., y Desobry, S. (2006). Encapsulación de sabor y liberación controlad, revisada en; International Journal of Food Science and Technology. 41: 1-21. 